\title{
State Estimation of Target Tracking Based on Improved Input Estimation Algorithm
}

\author{
Yi-Wei Chen \\ Department of Mechanical Engineering, Air Force Institute of Technology \\ No. 1, Julun Rd., Gangshan District, Kaohsiung City 82063, Taiwan (R.O.C.) \\ Corresponding Author: chenyiwei530@yahoo.com.tw
}

\begin{abstract}
The estimation effectiveness for tracking a maneuvering target depends on the accurate modeling of target motion and the performance of the estimator. In this paper, a three-dimensional motion equation of target flight route is proposed to describe the motion state of maneuvering target. An improved input estimation algorithm is developed to solve the problem of noise disturbance for obtaining the target state precisely. It utilizes the residual innovation and the fuzzy logic theory to design a fuzzy weighting factor to improve the traditional input estimation method. The theoretical development of tracking technique is verified by using several different filters and compared with the Singer model method for tracking a maneuvering target. The simulation results prove that the improved input estimator has the better estimation performance than the other filters, and the proposed method outperforms greatly the Singer model method. The proposed tracking technique suggest a feasible method to improve the tracking ability of the radar especially for the maneuvering target with violent movement and the improved input estimation algorithm has a satisfied estimation performance.
\end{abstract}

Keywords: maneuvering target, Kalman filter, input estimation; fuzzy logic.

\section{Introduction}

The maneuvering target tracking problems consist of the present and the future of target state, and the measurement data contains the noise disturbance. The accurate modeling of target motion is an important issue for the reliable estimation performance when a single filter is used for system identification. However, the accurate modeling of target motion may be difficult in practice due to the lack of knowledge about the system and the practical limitations imposed by computational complexities ${ }^{(1)}$. The common modeling methods for maneuvering targets are constant acceleration model (CA), constant velocity model $(\mathrm{CV})$, current statistical model and Singer model. These motion models are combined mainly with Kalman filter to treat the target tracking problems.

Maneuvering target tracking is an important problem complicated by the fact that the radar fail to directly detect the target acceleration. Kalman filter has been widely used to estimate the state of target, but the estimation performance may be seriously degraded to the high agility targets. Many approaches based on Kalman filter contain the early work of Singer, who augmented Kalman filter with the target acceleration equation represented by a first-order autoregressive process. The augmented filter tracks a maneuvering target closely but its performance degrades compared with a simple Kalman filter when the target moves at constant velocity and straight motion ${ }^{(2-4)}$. Many different maneuvering target tracking techniques after the Singer model are generally grouped into two types: One is to detect the target maneuver and then deal with it: for instance, the input estimation methods ${ }^{(5-9)}$,the variable state dimension approach ${ }^{(10)}$, and so on ${ }^{(11)}$. The other one is using multiple target models to describe the target motion state: for instance, the interactive multiple model (IMM) algorithms $^{(12-14)}$, the adaptive IMM algorithm ${ }^{(15)}$, and so on $^{(16-21)}$. The first approach is discussed mainly in this paper.

The target usually has the large input of acceleration during the moving process and Kalman filter without the input item can't obtain the precise estimation of target state. Input estimation is an effective method which detects the 
existence of target maneuvers and directly estimates the magnitude of the unknown maneuvers ${ }^{(3)}$. In this approach, Kalman filter is used alone during the non-maneuvering periods. When the target starts to maneuver, the magnitude of target acceleration is identified by the least-square estimation method and that are combined with Kalman filter to precisely compute the estimates of target state ${ }^{(5-7)}$. However, the estimation performance of traditional input method is not satisfied due to the assumption of constant input when a target moves with non-constant acceleration. Various modified techniques of input estimation were developed to solve this deficiency ${ }^{(3,8,9)}$. Tuan and Hou developed the theory of adaptive robust weighted function to construct a recursive least-square estimator of adaptive weighting factor and this method could improve the recursive least-square estimator to control the variation of system input ${ }^{(22)}$. Chen and Lee utilized the fuzzy logic theory to construct the fuzzy weighting factor to replace the weighting factor of least-square estimator and developed the intelligent fuzzy weighted input estimation. The proposed method could track the input signal rapidly and reduce the noise disturbance to improve the performance of estimator $^{(23)}$.

In this paper, the three-dimensional (3-D) motion equation of target flight route combined with the improved input estimator is utilized to solve the target tracking problems. The 3-D motion equation of target flight route is used to replace the common target motion models. Three different filters are used to verify the proposed tracking technique. The developed theory of improved input estimator is described carefully. The proposed tracking technique would be compared with the Singer model method for tracking a maneuvering target.

\section{Modeling of Target Motion}

The 3-D motion equation of target flight route in this study is utilized by the development of three-dimensional aiming point missile guidance law ${ }^{(24)}$. The target moves at constant velocity or acceleration in 3-D space and uses three angles (yaw, roll and pitch) to change the flight route. It is usable to describe the maneuvering target motion state. The 3-D motion equation of target flight route is explained briefly as follows:

(1) The origin of inertial coordinate is the position of radar system. From the inertial coordinate to the target coordinate, the transfer sequence of Euler angle is yaw
$\left(\psi_{T}\right) \rightarrow \operatorname{roll}\left(\phi_{T}\right) \rightarrow \operatorname{pitch}\left(\theta_{T}\right)$.

(2) The target is defined as a point-mass and the superscript $B$ represents the target coordinate. Fig. 1 is the target coordinate. The longitudinal direction of target is in $\hat{X}_{T}^{B}$ axis and the right direction of target is in $\hat{Y}_{T}^{B}$ axis. $\hat{Z}_{T}^{B}$ axis is perpendicular to $\hat{X}_{T}^{B}$ and $\hat{Y}_{T}^{B}$ axis.

(3) The target moves at the longitudinal acceleration $a_{T L}$ in $\hat{X}_{T}^{B}$ axis and the target uses the aileron to tilt the fuselage with the angle of roll $\left(\phi_{T}\right)$ to turn left or right to change the flight route, and makes the lateral movement or flee by the lateral acceleration $a_{T R}$ in $\hat{Z}_{T}^{B}$ axis.

(4) The longitudinal velocity anytime is $u_{T}^{B}(t)$. The target changes the flight route by using the roll angle and the lateral acceleration so both the velocity components in $\hat{Y}_{T}^{B}$ axis $\left(v_{T}^{B}\right)$ and $\hat{Z}_{T}^{B}$ axis $\left(w_{T}^{B}\right)$ are zero anytime.

(5) The initial longitudinal velocity of target, longitudinal acceleration, lateral acceleration, yaw, roll, pitch and the initial position in the inertial coordinate have been known or estimated.

The transformation matrix is defined as $[T]^{B / I}$ when the inertial coordinate is transferred to the target coordinate, and the transformation matrix $[T]^{I / B}$ is on the contrary. The transformation matrix elements are defined as follows:

$$
\begin{gathered}
{[T]^{B / I}=\left[\begin{array}{lll}
B_{11} & B_{12} & B_{13} \\
B_{21} & B_{22} & B_{23} \\
B_{31} & B_{32} & B_{33}
\end{array}\right]} \\
{[T]^{I / B}=\left[\begin{array}{lll}
B_{11} & B_{21} & B_{31} \\
B_{12} & B_{22} & B_{32} \\
B_{13} & B_{23} & B_{33}
\end{array}\right]}
\end{gathered}
$$

$B_{11}=\cos \theta \cos \psi-\sin \theta \sin \phi \sin \psi$

$B_{12}=\cos \theta \sin \psi+\sin \theta \sin \phi \cos \psi$

$B_{13}=-\sin \theta \cos \phi$

$B_{21}=-\cos \phi \sin \psi$

$B_{22}=\cos \phi \cos \psi$

$B_{23}=\sin \phi$

$B_{31}=\sin \theta \cos \psi+\cos \theta \sin \phi \sin \psi$

$B_{32}=\sin \theta \sin \psi-\cos \theta \sin \phi \cos \psi$

$B_{33}=\cos \theta \cos \phi$

The initial velocity components of target in the inertial coordinate are transferred to that in the target coordinate to 
obtain the initial longitudinal velocity $u_{T 0}^{B}$. Besides, there's only the velocity component $u_{T}^{B}(t)$ in $\hat{X}_{T}^{B}$ axis in the target coordinate.

$$
\begin{gathered}
{\left[\begin{array}{c}
u_{T 0}^{B} \\
v_{T 0}^{B} \\
w_{T 0}^{B}
\end{array}\right]=[T]^{B / I}\left[\begin{array}{c}
u_{T 0}^{I} \\
v_{T 0}^{I} \\
w_{T 0}^{I}
\end{array}\right]} \\
u_{T 0}^{B}=B_{11} u_{T 0}^{I}+B_{12} v_{T 0}^{I}+B_{13} w_{T 0}^{I}
\end{gathered}
$$

The longitudinal velocity at any time in the target coordinate is as follows:

$$
u_{T}^{B}(t)=u_{T 0}^{B}+a_{T L} t
$$

The velocity components at any time in the target coordinate are transferred to that in the inertial coordinate, and the differential of position is the velocity in the inertial coordinate.

$$
\begin{gathered}
{\left[\begin{array}{l}
x_{T}^{\prime I}(t) \\
y_{T}^{\prime I}(t) \\
z_{T}^{\prime I}(t)
\end{array}\right]=\left[\begin{array}{l}
u_{T}^{I}(t) \\
v_{T}^{I}(t) \\
w_{T}^{I}(t)
\end{array}\right]=[T]^{I / B}\left[\begin{array}{c}
u_{T}^{B}(t) \\
v_{T}^{B}(t) \\
w_{T}^{B}(t)
\end{array}\right]} \\
=u_{T}^{B}(t)\left[\begin{array}{c}
\cos \theta_{T} \cos \psi_{T}-\sin \theta_{T} \sin \phi_{T} \sin \psi_{T} \\
\cos \theta_{T} \sin \psi_{T}+\sin \theta_{T} \sin \phi_{T} \cos \psi_{T} \\
-\sin \theta_{T} \cos \phi_{T}
\end{array}\right]
\end{gathered}
$$

In addition, the angular velocity of target can be shown by the component of unit vector $l, m, n$ :

$$
\vec{\Omega}=l \hat{X}_{B}+m \hat{Y}_{B}+n \hat{Z}_{B}
$$

In the target coordinate, the target angular velocity is influenced by the lateral acceleration:

$$
\begin{aligned}
& l=0 \\
& m=a_{T R} / u_{T}^{B}(t) \\
& n=0
\end{aligned}
$$

The target angular velocity is transferred to Euler angular velocity:

$$
\begin{aligned}
& \psi_{T}^{\prime}(t)=\left(-l \sin \theta_{T}+n \cos \theta_{T}\right) / \cos \phi_{T}=0 \\
& \phi_{T}^{\prime}(t)=l \cos \theta_{T}+n \sin \theta_{T}=0 \\
& \theta_{T}^{\prime}(t)=-\dot{\psi}_{T} \sin \phi_{T}+m=a_{T R} / u_{T}^{B}(t)
\end{aligned}
$$

Therefore, the differential equations for the 3-D motion equation of target flight route are as follows:

$$
\left[\begin{array}{c}
u_{T}^{\prime B}(t) \\
\theta_{T}^{\prime}(t) \\
x_{T}^{\prime \prime}(t) \\
y_{T}^{\prime I}(t) \\
z_{T}^{\prime I}(t)
\end{array}\right]=\left[\begin{array}{c}
a_{T L} \\
a_{T R} / u_{T}^{B}(t) \\
u_{T}^{B}(t)\left[\cos \theta_{T}(t) \cos \psi_{T}(t)-\sin \theta_{T}(t) \sin \phi_{T}(t) \sin \psi_{T}(t)\right] \\
u_{T}^{B}(t)\left[\cos \theta_{T}(t) \sin \psi_{T}(t)-\sin \theta_{T}(t) \sin \phi_{T}(t) \cos \psi_{T}(t)\right] \\
-u_{T}^{B}(t) \sin \theta_{T}(t) \cos \phi_{T}(t)
\end{array}\right]
$$

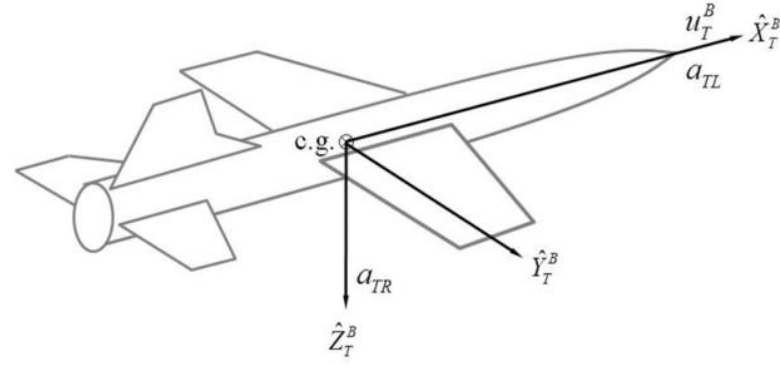

Fig. 1. Target coordinate.

\section{Filters}

\subsection{Extended Kalman Filter (EKF)}

Most of estimated systems are nonlinear in the world. EKF is Kalman filter in essence used for linearizing and discretizing the nonlinear system and has a wide application ${ }^{(25-27)}$. The 3-D motion equation of target flight route is a nonlinear continuous system. The proposed system in this study can be linearized and discretized through EKF and the algorithm is explained briefly as follows:

State and measurement equations:

$$
\begin{aligned}
& X(k)=f(X(k-1), u(k-1), w(k-1)) \\
& Z(k)=h(X(k), v(k))
\end{aligned}
$$

Kalman filer consists of five operation steps:

$$
\begin{gathered}
\hat{X}^{-}(k)=A \hat{X}(k-1)+B u(k-1) \\
P^{-}(k)=A P(k-1) A^{T}+Q \\
K(k)=P^{-}(k) H^{T}\left[H P^{-}(k) H^{T}+R\right]^{-1} \\
\hat{X}(k)=\hat{X}^{-}(k)+K(k)\left[Z(k)-h(k)\left(\hat{X}^{-}(k)\right)\right] \\
P(k)=(I-K(k) H) P^{-}(k)
\end{gathered}
$$

And

$$
\begin{aligned}
& A=I+\frac{\partial}{\partial X(k-1)} f(X(k-1), u(k-1)) \Delta t \\
& H=\frac{\partial}{\partial X(k)} h(X(k)) \\
& B=I \Delta t
\end{aligned}
$$

$X(k)$ : System state vector.

$\hat{X}(k)$ : System state prediction vector.

$P^{-}(k)$ : Prediction error covariance matrix.

$P(k)$ : Estimation error covariance matrix.

$f:$ Nonlinear system state transition function.

$u(k)$ : System control input vector. 
$w$ : Process noise vector defined as the white noise of covariance matrix $Q$ and the mean is zero.

$Z(k)$ : System measurement vector.

$h$ : Nonlinear system measurement function.

$v$ : Measurement noise vector defined as the white noise of covariance matrix $R$ and the mean is zero.

From the Eq. (13), the precise estimation of Kalman filter is dependent on the system input $u$. Generally speaking, Kalman filter without the input term is usually used for the most of estimated system when the system input is unknown, and this condition easily leads to produce more estimation errors.

\subsection{Adaptive Weighting Input Estimation (AWIE)}

Tuan and Hou successfully combined Kalman filter with the recursive least-square estimator of adaptive weighting factor to improve the estimator for controlling the change of system input ${ }^{(22)}$. The proposed method can estimate the system state effectively and the algorithm is explained summarily as follows:

The linear discrete system state and measurement equations:

$$
\begin{aligned}
& X(k)=A X(k-1)+B u(k-1)+w(k-1) \\
& Z(k)=H X(k)+v(k)
\end{aligned}
$$

Use Kalman filter without the input item:

$$
\begin{gathered}
\bar{X}^{-}(k)=A \bar{X}(k-1) \\
P^{-}(k)=A P(k-1) A^{T}+Q \\
s(k)=H P^{-}(k) H^{T}+R \\
K_{a}=P^{-}(k) H^{T} s^{-1}(k) \\
P(k)=\left(I-K_{a} H\right) P^{-}(k) \\
\bar{Z}(k)=Z(k)-H \bar{X}^{-}(k) \\
\bar{X}(k)=\bar{X}^{-}(k)+K_{a} \bar{Z}(k)
\end{gathered}
$$

Combined with the recursive least-square estimator:

$$
\begin{gathered}
N(k)=H[A M(k-1)+I] B \\
M(k)=\left[I-K_{a} H\right][A M(k-1)+I] \\
K_{b}=\gamma^{-1} P^{\prime}(k-1) N^{T}(k)\left[N(k) \gamma^{-1} P^{\prime}(k-1) N^{T}(k)+s(k)\right]^{-1} \\
P^{\prime}(k)=\left[I-K_{b} N(k)\right] \gamma^{-1} P^{\prime}(k-1) \\
\hat{u}(k)=\hat{u}(k-1)+K_{b}[\bar{Z}(k)-N(k) \hat{u}(k-1)]
\end{gathered}
$$

$P^{\prime}(k)$ : Input estimation error covariance matrix.

$K_{a}$ : Kalman gain.

$K_{b}$ : Steady-state correction gain.

$M(k), N(k):$ Sensitivity matrix.

$\gamma$ : Weighting factor. $s(k)$ : Innovation covariance vector.

$\bar{Z}(k)$ : Residual innovation vector.

The recursive least-square estimator is mainly used to compute the input estimation vector and that could be utilized by the Kalman filter with the input item at the next recursive step to obtain the more precise estimation of system state. Besides, the recursive least-square estimator with the weighting factor has both function of smoothing and forgetting. It is because the weighting factor would lead the recursive least-square estimator to forget the old information and the least-square method can smooth the information. The weighting factor is designed in the interval $(0,1)$. It would be the traditional recursive least-square estimator applied to the estimation of steady parameters if the weighting factor is 1 . The weighting factor determines the forgetting validity of the estimator. $K_{b}$ would get larger as $\gamma$ gets smaller in terms of the Eq. (29). The forgetting validity would be more apparent in terms of the Eq. (30). The higher the forgetting validity is, the lower the smoothing validity would be. When the system state changes a lot, the latest information would be very significant and the recursive least-square estimator needs the faster forgetting speed to treat the old information. For solving the problem, Tuan and Hou developed the theory of adaptive robust weighting function $\gamma(k)$ and it is used to replace $\gamma$ in Eq. (29) and Eq. (30) to obtain the better estimation performance. The function equation is defined as follows:

$$
\gamma(k)=\left\{\begin{array}{c}
1, \quad S(k) \leq \mu \\
\frac{\mu}{S(k)}, S(k)>\mu
\end{array}\right.
$$

$\mu$ is the deviation of measurement noise covariance and $S(k)$ is the modulus of the residual innovation vector.

\subsection{Improved Input Estimator (IIE)}

According to the above description, the weighting factor of AWIE shown in Equation (32) is revised by $S(k)$ and $\mu$. Therefore, $S(k)$ would get larger when the unknown input prompts variation and the weighting function would get smaller if $S(k)>\mu$. The recursive least-square estimator would accelerate the tracking speed and it is easy to produce the larger oscillation during the estimation process if the system state changes violently. On the contrary, $S(k)$ would get smaller when the unknown input has smaller variation and the weighting function 
would be larger or 1 . According to the above reasons, the adaptive weighting function leads the recursive least-square estimator to produce the oscillation easily and the estimator is unable to estimate the unknown input steadily. It needs to choose the suitable weighting factor to obtain the ideal estimator according to the variation of system parameters. Chen and Lee utilized the fuzzy logic theory to construct the fuzzy weighting factor to replace the weighting factor of least-square estimator for improving this condition ${ }^{(23)}$. It is useful to track the input signal rapidly without producing the huge oscillation. However, the design for the input of fuzzy logic system is sensitive to the signal input. The divergence may produce when the system state changes violently such as the moving aircraft with strong maneuver.

In this paper, an improved input estimator is developed to solve the problems above and estimate the target state precisely. In this algorithm, the residual innovation is utilized to be the evaluation criteria for choosing the suitable weighting factor. During the estimation process, the weighting factor would be 1 if $S(k) \leq \mu$. The system state changes violently because of the unknown input $(S(k)>\mu)$, the fuzzy logic theory is utilized to develop a fuzzy weighting factor which can accelerate the tracking speed of the estimator steadily without producing the oscillation. The fuzzy logic system consists of fuzzy rules base, fuzzy inference engine, fuzzifier and defuzzifier. The input of fuzzy logic system is designed mainly to control the change produced by the unknown input as follows:

$$
\psi(k)=\frac{\mu}{S(k)}
$$

The input of fuzzy logic system is used to modify the weighting factor to construct the fuzzy weighted estimator. The variables of fuzzy logic system contain both the input $\psi(k)$ and the output $\gamma(k)$, and the values are defined in the interval $(0,1) . A$ and $B$ are both fuzzy sets of $\psi(k)$ and $\gamma(k)$, and that are labeled in the linguistic terms of VL (Very Large), L (Large), M (Medium), S (Small), VS (Very Small). The fuzzy membership function is defined by S-shaped curve function shown in Fig. 2. The Mamdani maximum-minimum inference engine is utilized and the center of gravity method is the algorithm of the defuzzifier. The fuzzy weighting factor would be computed by the operation of fuzzy logic system. Fig. 3 is the flow chart of improved input estimator. The fuzzy rules base used in this theory is the collection of IF-THEN rules as follows:

Rule 1: If $\psi(k)$ is VS, then $\gamma(k)$ is VS;

Rule 2: If $\psi(k)$ is S, then $\gamma(k)$ is S;

Rule 3: If $\psi(k)$ is $\mathrm{M}$, then $\gamma(k)$ is $\mathrm{M}$;
Rule 4: If $\psi(k)$ is $\mathrm{L}$, then $\gamma(k)$ is $\mathrm{L}$;

Rule 5: If $\psi(k)$ is VL, then $\gamma(k)$ is VL.

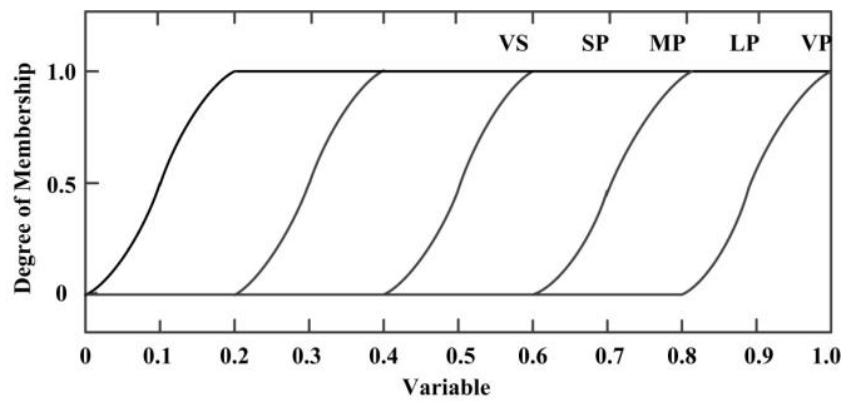

Fig. 2. S-Shaped membership function.

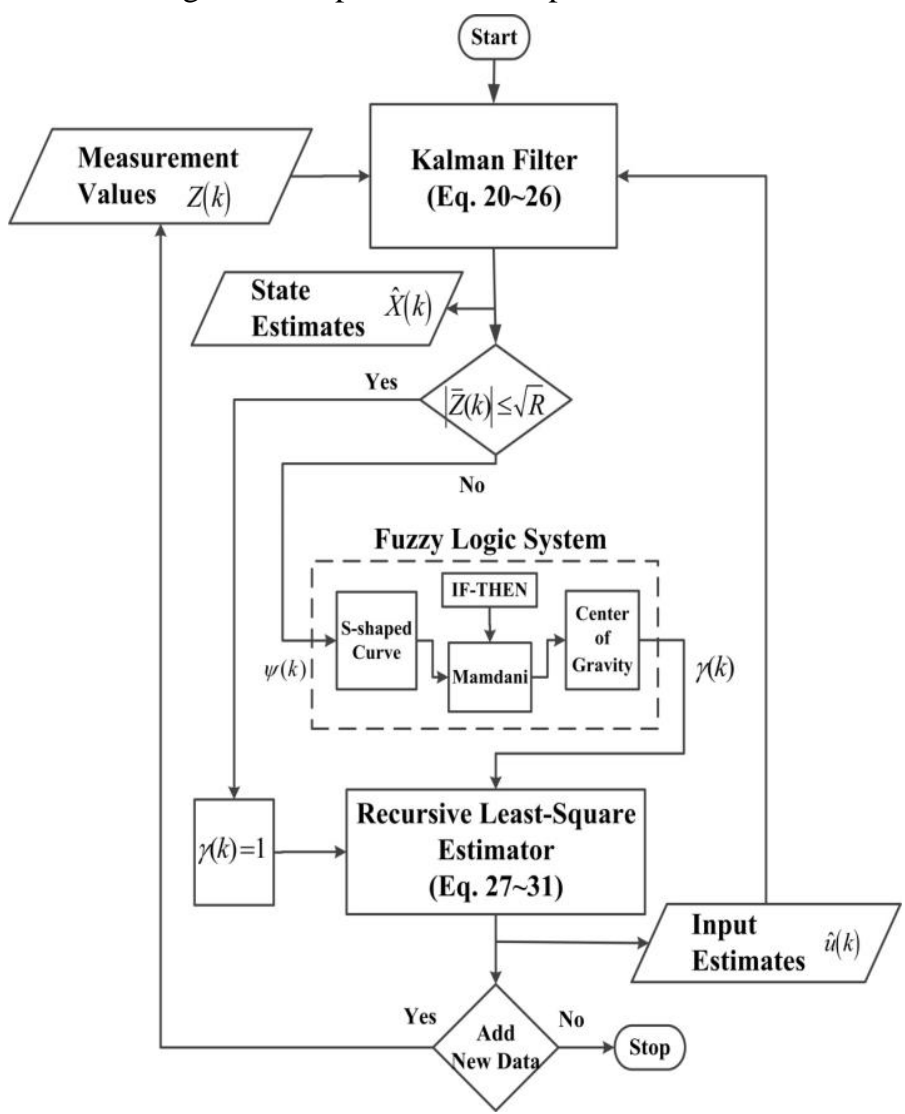

Fig. 3. Flow chart of improved input estimator.

\section{Results and Discussion}

The proposed tracking technique for a maneuvering target in this paper is verified by using the Kalman filter (KF), AWIE, IIE, and compared with the Singer model method. It is assumed the target moves in a 3-D space and the motion state includes the constant velocity, acceleration, deceleration, turning, diving and climbing. Fig. 4 is the target flight route in the inertial coordinate and the parameters of simulation are designed as follows:

(1) Flight time (sec): 30.

(2) Initial position (m): $(5000,5000,5000)$.

(3) Initial velocity $(\mathrm{m} / \mathrm{sec}): 250$. 
(4) Initial angle of yaw, roll, pitch (degree): 15, 0, 0. The roll angle changes to 120 in 5 10 seconds; -60 in 25 30 seconds.

(5) Longitudinal acceleration $\left(\mathrm{m} / \mathrm{sec}^{2}\right): 10$ in 5 10 seconds; 20 in 15 18 seconds; -10 in 25 30 seconds.

(6) Lateral acceleration $\left(\mathrm{m} / \mathrm{sec}^{2}\right)$ : -30 in 5 10 seconds; -10 in $15 \sim 18$ seconds.

(7) Process noise covariance $Q=10^{-2}$ and measurement noise covariance $R=100$.

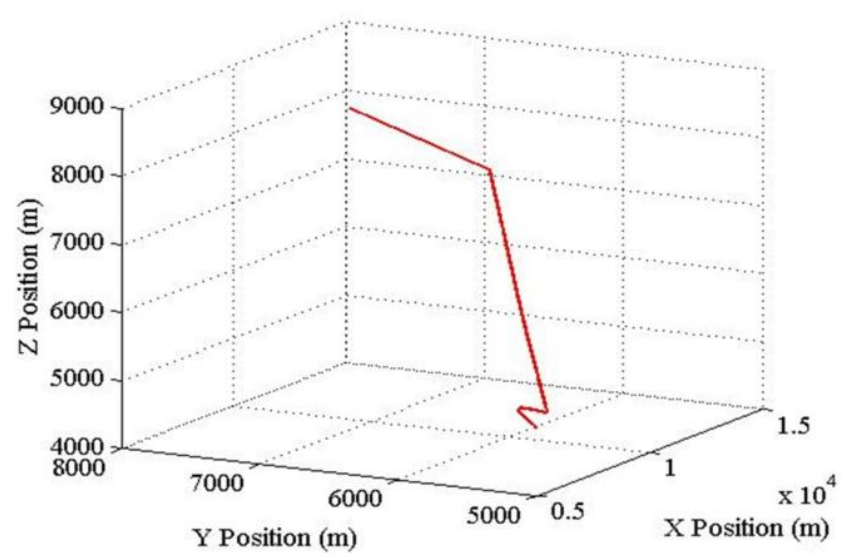

Fig. 4. Target flight route.

The target dynamic movement is drawn easily by using the 3-D motion equation of target flight route, but it may be difficult for the common target motion equation such as the constant acceleration motion equation applied on the simulation of target tracking problems.

Fig. 5 and Fig. 6 are the truth and estimation of three different filters in longitudinal acceleration and lateral acceleration respectively. IIE and AWIE can track the acceleration of target rapidly compared with $\mathrm{KF}$ when the target has the acceleration input. However, AWIE would easily produce the oscillation during the estimation process shown in 0 5 seconds of Fig. 5.

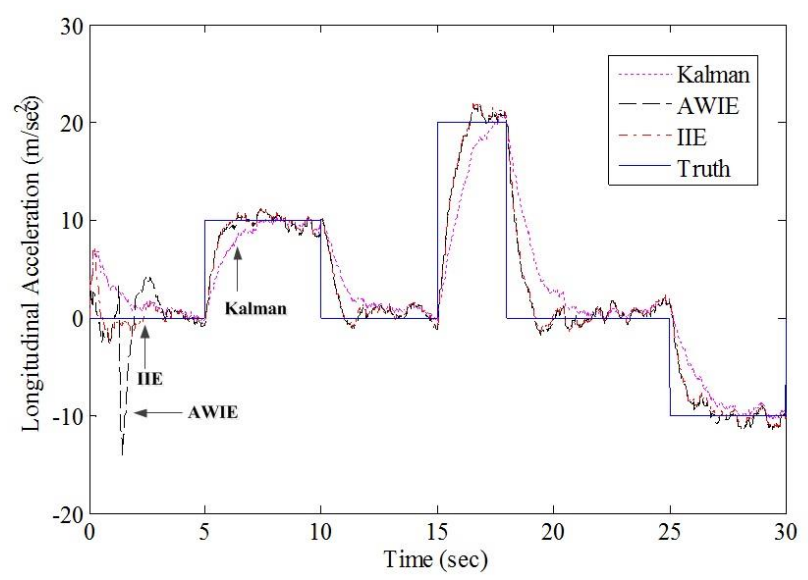

Fig. 5. Estimation of three different filters in longitudinal acceleration.

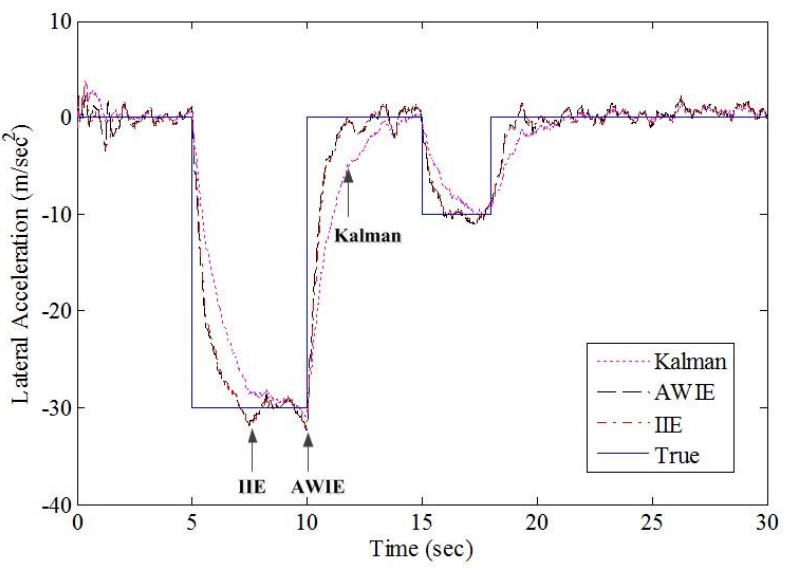

Fig. 6. Estimation of three different filters in lateral acceleration.

Singer proposed a target acceleration model which is the first model that characterizes the unknown acceleration of target as a time correlation random process and has been the basis for developing the maneuvering target models. Singer model combined with Kalman filter can estimate the motion state of maneuvering target effectively. The state equation of Singer model is in Eq. (34) and the algorithm is introduced carefully in Reference ${ }^{(4)}$.

$$
\dot{X}(t)=\left[\begin{array}{ccc}
0 & 1 & 0 \\
0 & 0 & 1 \\
0 & 0 & -\alpha
\end{array}\right] X(t)+\left[\begin{array}{l}
0 \\
0 \\
1
\end{array}\right] \omega(t)
$$

$\alpha$ : Maneuvering frequency.

$\omega(t)$ : Process noise.

In this paper, Singer model method is used to compared with the proposed tracking technique and the parameters of simulation are assumed as $P_{0}=0.5, \quad P_{\max }=0.1$, $a_{\max }=\left[\begin{array}{lll}30 & 30 & 30\end{array}\right]\left(\mathrm{m} / \mathrm{sec}^{2}\right), \quad \alpha=1 / 10$.

$P_{0}$ : Probability for the target may move without the acceleration.

$P_{\max }$ : Probability for the target may accelerate or decelerate at a maximum rate $a_{\max }$.

Fig. 7-9 are the estimation errors of three different filters and Singer model in $\mathrm{X}, \mathrm{Y}, \mathrm{Z}$ position. It is obvious the estimation error of Singer model is close to the proposed tracking technique but the estimation error increases quickly when the target starts to change the motion state after 5 th second. The reason may come from that the Singer model is not suitable for the maneuvering target with violent movement. The proposed tracking technique which uses three different filters outperforms greatly the Singer model method. IIE and AWIE has the better estimation precision than KF, but AWIE is easy to produce the unsteady condition shown in 5 10 seconds of 
Fig. 7, 9. KF has the worst estimation precision among three different filters. As a result, the proposed tracking technique based on IIE has the better estimation performance. IIE can track the input signal rapidly and reduce the noise disturbance effectively for obtaining the precise estimates of target motion state.

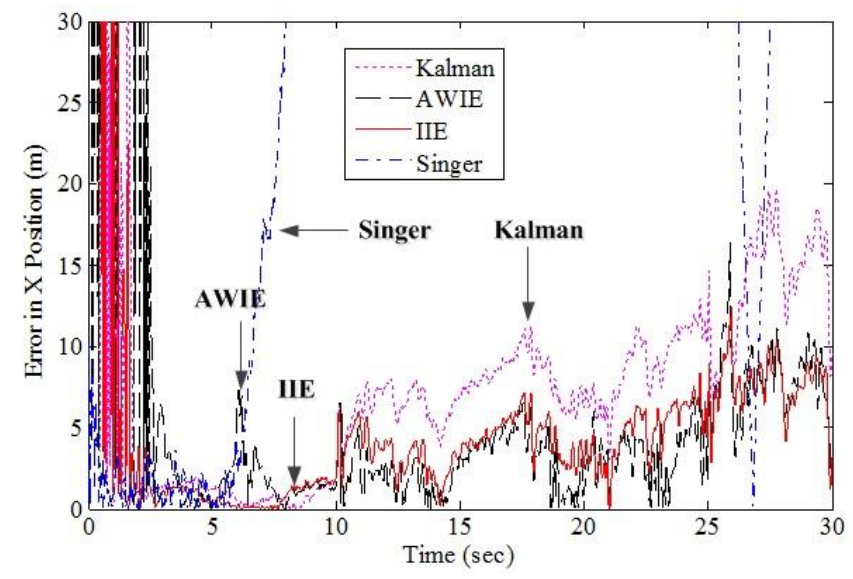

Fig. 7. Error in X position.

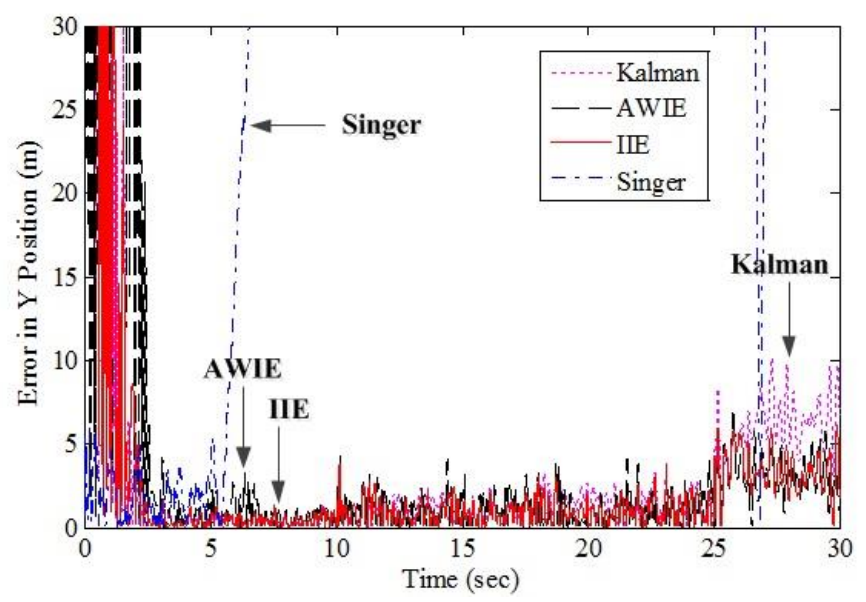

Fig. 8. Error in Y position.

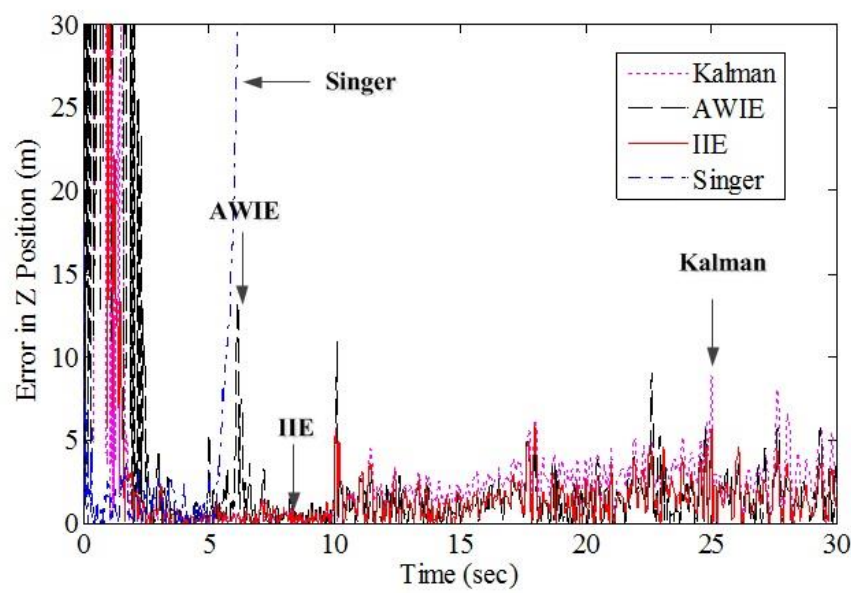

Fig. 9. Error in $\mathrm{Z}$ position.

In this paper, the proposed target tracking technique absolutely has the satisfied performance for the state estimation of target tracking. However, the 3-D motion equation of target flight route combined with IIE would need faster computation speed for the requirement of computer hardware than the common tracking techniques.

\section{Conclusions}

IIE is developed based on the input estimation method and the fuzzy logic system for inferring the weighting factor. It is used to estimate the maneuvering target motion state and has the comparison with KF and AWIE. A feasible tracking technique for a maneuvering target is proposed in this paper. The proposed method utilizes the 3-D motion equation of target flight route for the accurate modeling of maneuvering target motion and uses three different filters to verify the theoretical development, and that are also compared with the Singer model method. The simulation results prove that the proposed tracking technique can estimate the maneuvering target with violent movement effectively, and IIE has the better estimation performance than KF and AWIE. It can not only track the input signal rapidly but also have the high stability during the estimation process. The proposed tracking technique has a high performance and effectiveness for the state estimation of maneuvering target.

\section{References}

(1) J.H. Ryu, D.H. Han, K.K. Lee, and T.L. Song, "Prediction-based interacting multiple model estimation algorithm for target tracking with large sampling periods", International Journal of Control, Automation, and Systems, Vol. 6, No. 1, pp. 44-53, 2008.

(2) B.-J. Lee, Y.-H. Joo, and J. B. Park, "An intelligent tracking method for a maneuvering target," International Journal of Control, Automation, and Systems, Vol. 1, No. 1, pp. 93-100, 2003.

(3) H. Khaloozadeh and A. Karsaz, "Modified input estimation technique for tracking manoeuvring targets," IET Radar Sonar Navigation, Vol. 3, No. 1, pp. 30-41, 2009.

(4) R. A. Singer, "Estimating optimal tracking filter performance for manned maneuvering targets," IEEE Transactions on Aerospace and Electronic System, AES-6, pp. 473-483, 1970.

(5) Y.T. Chan, A.G.C. Hu, and J.B. Plant, “A Kalman filter 
based tracking scheme with input estimation," IEEE Transactions on Aerospace and Electronic System, AES-15, No. 2, 237-244, 1979.

(6) P.L. Bogler, "Tracking a maneuvering target using input estimation", Transactions on Aerospace and Electronic System, AES-23, No. 3, 298-310, 1987.

(7) Y.T. Chan and F. Couture, "Maneuver detection and tracking correction by input estimation", IEE Proceedings, Vol. 140, No. 1, pp. 21-28, 1993.

(8) H.I. Whang, J.G. Lee, and T.K. Sung, "Modified input estimation technique using pseudoresiduals", IEEE Transactions on Aerospace and Electronic System, Vol. 30, No. 1, pp. 220-228, 1994.

(9) H. Lee and M.J. Tahk, "Generalized input-estimation technique for tracking maneuvering targets", IEEE Transactions on Aerospace and Electronic System, Vol. 35, No. 4, pp. 1388-1402, 1999.

(10) Y. Bar-shalom and K. Birmiwal, "Variable dimension filter for maneuvering target tracking", IEEE Transactions on Aerospace and Electronic System, AES-18, Vol. 5, pp. 621-629, 1982.

(11) Y.H. Park, J.H. Seo, and J.G. Lee, "Tracking using the variable-dimension filter with input estimation", IEEE Transactions on Aerospace and Electronic System, Vol. 31, No. 1, pp. 399-408, 1995.

(12)H.A.P. Blom and Y. Bar-Shalom, "The interacting multiple model algorithm for systems with a jump-linear smoothing application", IEEE Transactions on Automatic Control, Vol. 33, No. 8, pp. 780-783, 1988.

(13) E. Mazor, A. Averbuch, Y. Bar-Shalom, and J. Dayan, "Interacting multiple model methods in target tracking: A survey", Transactions on Aerospace and Electronic System, AES-6, No. 4, pp. 473-483, 1993.

(14) Y. Bar-shalom, K.C. Chan, and H.A.P. Blom, "Tracking a maneuvering target using input estimation versus the interacting multiple model algorithm", IEEE Transactions on Aerospace and Electronic System, Vol. 25, No. 2, pp. 296-300, 1989.

(15)A. Munir and D.P. Atherton, "Adaptive interacting multiple model algorithm for tracking a maneuvering target", IEE Proceedings-Radar, Sonar and Navigation, Vol. 142, No. 1, pp. 11-17, 1995.

(16)B.J. Lee, J.B. Park, H.J. Lee, and Y.H. Joo, "Fuzzy-logic-based IMM algorithm for tracking a manoeuvring target", IEE Proceedings-Radar, Sonar and Navigation, Vol. 152, No. 1, pp. 16-22, 2005.

(17) B.J. Lee, Y.H. Joo, and J.B. Park, "An intelligent tracking method for a maneuvering target", International Journal of Control, Automation and Systems, Vol. 1, No. 1, pp. 93-100, 2003.

(18) I. Turkmen, "IMM fuzzy probabilistic data association algorithm for tracking maneuvering target", Expert Systems with Applications, No. 34, pp. 1243-1249, 2008.

(19) D. Mohammed, K. Mokhtar, and O. Abdelaziz, "A new IMM algorithm using fixed coefficients filters (fastIMM)", AEU-International Journal of Electronics and Communications, No. 64, pp. 1123-1127, 2010.

(20) P.C. Tuan and L.W. Fong, "IMM tracking algorithm with input estimation", International Journal of Systems Science, Vol. 27, No. 7, pp. 629-639, 1996.

(21) Y.L. Lee and Y.W. Chen, "IMM estimator based on weighted input estimation for tracking a maneuvering target", Applied Mathematical Modelling, No. 39, pp. 5791-5802, 2015.

(22) P.C. Tuan and W.T. Hou, "Adaptive robust weighting input estimation method for the 1-D inverse heat conduction problem", Numerical Heat Transfer Part B-Fundamentals, No. 34, pp. 1-18, 1998.

(23) T.C. Chen and M.H. Lee, "Intelligent fuzzy weighted input estimation method applied to inverse heat conduction problems", International Journal of Heat and Mass Transfer, Vol. 58, No. 6, pp. 4168-4183, 2008.

(24) Y.P. Lin, L.P. Tsao, and C.L. Lin, "Development of three-dimensional aiming point guidance law", International Journal of Systems Science, Vol. 41, No. 11, pp. 1353-1362, 2010.

(25) Y. Chinniah, R. Burton, S. Habibi, and E. Sampson, "Identification of the nonlinear friction characteristics in a hydraulic actuator using the extended kalman filter", Transactions of the Canadian Society for Mechanical Engineering, Vol. 32, No. 2, pp. 121-136, 2008.

(26) S. Wang, S. Habibi, and B. Richard, "A comparative study of a smooth variable structure filter and the extended kalman filter", Transactions of the Canadian Society for Mechanical Engineering, Vol. 32, No. 3-4, pp. 353-370, 2008.

(27) M.W. Owen, and A.R. Stubberud, “A Neural extended kalman filter multiple model tracker", OCEANS 2003 Proc. 4, pp. 2111-2119, 2003. 